\title{
Risk Evaluation of Debris Flow Hazard Based on Asymmetric Connection Cloud Model
}

\author{
Xinyu Xu, Mingwu Wang, Yafeng Li, and Libiao Zhang \\ School of Civil and Hydraulic Engineering, Hefei University of Technology, Hefei 230009, China \\ Correspondence should be addressed to Mingwu Wang; wanglab307@foxmail.com
}

Received 14 February 2017; Revised 21 August 2017; Accepted 12 September 2017; Published 26 October 2017

Academic Editor: Marco Pizzarelli

Copyright (C) 2017 Xinyu Xu et al. This is an open access article distributed under the Creative Commons Attribution License, which permits unrestricted use, distribution, and reproduction in any medium, provided the original work is properly cited.

\begin{abstract}
Risk assessment of debris flow is a complex problem involving various uncertainty factors. Herein, a novel asymmetric cloud model coupled with connection number was described here to take into account the fuzziness and conversion situation of classification boundary and interval nature of evaluation indicators for risk assessment of debris flow hazard. In the model, according to the classification standard, the interval lengths of each indicator were first specified to determine the digital characteristic of connection cloud at different levels. Then the asymmetric connection clouds in finite intervals were simulated to analyze the certainty degree of measured indicator to each evaluation standard. Next, the integrated certainty degree to each grade was calculated with corresponding indicator weight, and the risk grade of debris flow was determined by the maximum integrated certainty degree. Finally, a case study and comparison with other methods were conducted to confirm the reliability and validity of the proposed model. The result shows that this model overcomes the defect of the conventional cloud model and also converts the infinite interval of indicators distribution into finite interval, which makes the evaluation result more reasonable.
\end{abstract}

\section{Introduction}

Debris flow is a common natural hazard that frequently appears in the mountainous region of southwestern China, such as Gongshan district and Dongchuan district in Yunnan province, and Ridigou district in Sichuan province. Its occurrence is difficult to accurately predict due to complex nature and outbreaks of sudden, ferocious, and swift characteristics [1]. Debris flow often results in heavy casualties and property losses and brings great harm to the state of regional development and public safety, so it is a serious impediment to social and economic development in mountain areas. Consequently, risk assessment of debris flow has been a major concern for forecasting and prevention work.

Systematic observation and research on debris flows have been carried out since the 1970s [1]. Generally, the hazard grade of debris flow, which is defined as the magnitude and frequency of occurrence of debris flow, is used to assess the risk of debris flow, but it involves many uncertainty factors including internal and environmental factors [2]. Thus, sometimes the single attribute method or simple methods cannot meet the requirements of actual conditions and did not produce reliable and stable results. So researchers endeavored to present various methods for the rational prediction of debris flow, such as grey relational analysis method [3], extension method $[4,5]$, fuzzy comprehensive evaluation method [6], and BP neural network method [7], and obtained many useful results. And geographic information system (GIS) technology has also been introduced into the assessment of debris flows in recent years $[8,9]$. On the other hand, those methods were of their own disadvantages. For the grey relational analysis method, rational results might not be obtained due to the fluctuation of the evaluation index value. Some constraint conditions might be missed in extension method; it would lead to the deviation of evaluation results. Fuzzy set method was often affected by the rational definition of membership function. The major shortcoming of BP neural network method was its inability to present an explicit relationship between debris flow and evaluation parameters with rank potential and its application was limited by knowledge acquisition. The mapping approach which used GIS technology might be arbitrarily different in the discrete risk assessment of debris flow system. Overall, whereas these researches have advanced the risk assessment of debris flow, it 
is still not well resolved nowadays since debris flow evaluation involves various uncertainty influence factors. The newly developed cloud model theory shows its advantage in dealing with the uncertainty problems [10]; the certainty degree of the measured sample to classification grades is generated automatically by the special algorithm, which avoids the defect of determining the certainty degree subjectively and considers the fuzziness and randomness of evaluation indicators as a whole and achieves the qualitative and quantitative conversion, so it provides a new idea for assessment risk of debris flow. However, there is an obvious defect in the conventional cloud model that indicators must obey normal distribution and locate in infinite interval; this is not always in accordance with the actual indicators distribution, which inevitably limits its application.

The main objective of this paper is to introduce the asymmetric connection cloud method based on coupling set pair analysis and cloud theory for the evaluation of debris flow hazard risk and conquer the application limitation of conventional normal cloud model. The proposed method can describe dialectically the certainty and uncertainty relationships between the measured evaluation indicators and each classification standard in a unified way and also expresses conversion tendency of classification boundary. Moreover, a practical example is given to investigate the feasibility and effectiveness of this method.

\section{Theory}

2.1. Cloud Model. Cloud theory put forward by Dr. Li [10] is a kind of conversion model between nature variable and quantity variable based on the probability theory and fuzzy set theory. It is widely adopted to analyze the engineering problems with characteristics of fuzziness and randomness. The production of cloud drop is the key content of cloud model since the certainty degree of cloud drop can embody the fuzziness and randomness of uncertainty problem, which can quantitatively describe the qualitative concept from a unified perspective. The conventional algorithm of normal cloud is defined as follows: denote $\mathbf{C}$ as a qualitative concept in quantitative domain $\mathrm{Y}$. Quantization value $x \in \mathrm{Y}$ is a random realization of concept $\mathbf{C}$ in domain Y. Denote $\mu(x) \in[0,1]$ as the certainty degree of $x$ representing qualitative concept $\mathbf{C}$. $\mu(x)$ is a random number with stable tendency. There is $\mu$ : $Y \rightarrow[0,1], \forall x \in Y, x \rightarrow \mu(x)$. The distribution of $x$ in quantitative domain $\mathrm{Y}$ is called a cloud; each $x$ is called a cloud drop. If $x \sim N\left(E_{x}, E_{n}^{\prime 2}\right), E_{n}^{\prime} \sim N\left(E_{n}, H_{e}^{2}\right)$, the certainty degree of $x$ is calculated with

$$
\mu=\exp \left(\frac{-\left(x-E_{x}\right)^{2}}{2 E_{n}^{\prime 2}}\right),
$$

where $E_{x}, E_{n}$, and $H_{e}^{2}$, are expected value, entropy, and hyper entropy. However, the obtained normal cloud mapping is in an infinite interval, has an inability to reflect the characteristic of evaluation indicators in a finite interval, and neglects the uncertainty relationship and conversion tendency between the classification grades, which makes the evaluation results deviate from the actual situation and makes invalid for forecasting and prevention of debris flow. To overcome these shortcomings of the conventional normal cloud model, the identical, discrepancy and contrary (IDC) concept based on set pair theory was introduced to improve the normal cloud and deal with the certainty and uncertainty relationship between the risk grades and evaluation factors.

2.2. Set Pair Analysis. Set pair theory is a novel analytical method for systematic problems of uncertainty [11-16]. Both the dialectical law of the unity of opposites in philosophy and principle of universal connection are given consideration to depict uncertainty problems; the corresponding mathematical model is

$$
\mu=a+b I+c J
$$

where $\mu$ denotes the quantitative expression for set pair relationship, $a, b$, and $c$ are identity degree, discrepancy degree, and contrary degree, respectively, and $a+b+c=1$. $I$ is the discrepancy coefficient within $[-1,1] . J$ is the contrary coefficient and generally specified as -1 . Obviously, uncertainty and certainty of interaction and transformation can be treated dialectically by IDC analysis as a whole in set pair theory, which can comprehensively describe the complexity and diversity of dynamic uncertainty problem.

\section{Development of Asymmetric Connection Cloud}

3.1. Asymmetric Connection Cloud Model. The asymmetric connection cloud can be defined as follows: denote $\mathbf{C}$ as a qualitative concept in quantitative domain $\mathbf{Y}$, quantization value $x \in \mathrm{Y}$ is a random realization of concept $C$ in domain $\mathbf{Y}$, and the certainty degree of $x$ is

$$
\mu=\left[1-\left(\frac{x-E_{x}}{a^{\prime}}\right)^{2}\right]^{k} .
$$

Assume that the classification standard can be divided into $m$ evaluation grades $(i=1,2, \ldots, m)$, each grade has $n$ evaluation indexes $(j=1,2, \ldots, n)$, the cloud mapping of grade $i$ for index $j$ consists of both left and right half of asymmetric connection cloud with the boundary of expected value $E_{x}^{i}$, and cloud drop is obtained by the digital feature values $\left(E_{x}, E_{n}, H e, a^{\prime}, k\right)$ and the number of cloud drop $N$, which can be calculated by

$$
\begin{aligned}
E_{x}^{i} & =\frac{L_{\text {max }}^{i}+L_{\text {min }}^{i}}{2}, \\
E_{n}^{i} & =\frac{a_{i}}{\sqrt{2 k_{i}+3}}, \\
H_{e}^{i} & =\beta \cdot E_{n}^{i}, \\
a_{i}^{\prime} & =E_{n}^{i \prime} \cdot \sqrt{2 k_{i}+3}, \\
k_{i} & =\frac{\lg 0.5}{\lg \left[1-\left(\left(y_{i}-E_{x}^{i}\right) / a_{i}\right)^{2}\right]},
\end{aligned}
$$


where $E_{x}^{i}, E_{n}^{i}$, and $H_{e}^{i}$ are expected value, entropy, and hyper entropy of left interval or right interval of grade $i$ in asymmetric connection cloud, respectively; $a_{i}$ and $a_{i}^{\prime}$ are half-interval length and modified half-interval length; $x_{i}$ locates in interval $\left[E_{x}^{i}-a_{i}^{\prime}, E_{x}^{i}\right]$ when $a_{i}^{\prime}$ denotes modified left half- interval length; while $x_{i}$ locates in interval $\left[E_{x}^{i}, E_{x}^{i}+a_{i}^{\prime}\right]$ corresponding to $a_{i}^{\prime}$ denoting for the modified right half-interval length, in which $E_{n}^{i \prime}$ is generated by normal distribution, $E_{n}^{i \prime} \sim$ Normrnd $\left(E_{n}^{i}, H_{e}^{i 2}\right) . L_{\max }^{i}$ and $L_{\text {min }}^{i}$ are, respectively, the upper limitation and lower limitation of interval in the $i$ th evaluation grade. $\beta$ is atomization parameter and amended by the fuzzy degree; $\beta=0.01$ in this paper. $k_{i}$ is the order of corresponding distribution density function.

To quantitatively describe the transform tendency for evaluation results in each classification grade, the concept of certainty degree is adopted to analyze the possibility for index value in each class, and define the certainty degree of the boundary value of indicator interval for grade $i$ in grade $i$ and $i-1$ or $i+1$ as the same. So the possibility of the boundary value of indicator interval in grade $i$ equals that not in grade $i$; then the discrepancy degree in set pair analysis has an ability to describe this sort of uncertainty relationship and depict the transform tendency for identical degree or contrary degree. Therefore, the acquired certainty degree in $[0.5,1]$ based on asymmetric connection cloud can be defined as identical; the certainty degree in $(0,0.5]$ is defined as discrepancy; the certainty degree of 0 can be defined as contrary.

Actually, the asymmetric connection cloud model can take indictors distribution characteristic into consideration in the process of evaluation since the boundary interval and middle interval often show different distribution types, such that the distribution of the half-interval in boundary interval faring away the middle interval does not always obey normal distribution, while it obeys uniform distribution with the certainty degree for 1 . Besides, the conventional cloud model has an inability to deal with the evaluation index interval with the formation $\left[L_{\min },+\infty\right]$ or $\left[-\infty, L_{\max }\right]$; also the boundary intervals belong to asymmetric distribution, while the $L_{\text {mid }}$ value based on connection cloud model can be determined by both the left or right half-interval length and the symmetric point $E_{x}$ and denotes the upper limitation or lower limitation for the asymmetric interval. The evaluation index value shows monotonic increasing formation; the upper limitation value in the $m$ grade is determined by the following equation. Then, the certainty degree can be calculated by the above formula.

$$
L_{\text {mid }}=E_{x}^{m-1}+\left(E_{x}^{m-1}-L_{\min }^{m-2}\right) .
$$

3.2. Evaluation Procedures. The evaluation procedure with the model of asymmetric connection cloud is as follows.

First, the length of left half-interval $a_{i-L}$ and the length of right half-interval $a_{i-R}$ are calculated according to each grade of evaluation index standard.

Then, the corresponding digital feature values in left and right half-interval are determined.

Next, random number in a finite interval based on $M A T L A B$ program is obtained and the asymmetric cloud model of each evaluation index in various classification grades is simulated.

Finally, certainty degree of evaluation indicators in each class of evaluation sample is given, and combine indicator weights to calculate the integrated certainty degree.

Therefore, risk grade of evaluation sample is determined by the maximum integrated certainty degree.

3.3. Evaluation Model. Based on asymmetric connection cloud model, middle cloud and boundary cloud are obviously different. It might be a uniform distribution with certainty degree for 1 , when the measured value of evaluation indicator in boundary interval was far away from the middle interval. Otherwise, the certainty degree $\mu_{p, i j}$ to the grade $i$ for the $j$ th index of sample $p$ is calculated by

$$
\mu_{p, i j}=\left[1-\left(\frac{x_{0}-E_{x}^{i}}{a_{i}^{\prime}}\right)^{2}\right]^{k_{i}} .
$$

There are two types of evaluation indicators; for the first type of indicator which is defined for maximum - optimum index, corresponding lengths of left half-interval and right half-interval are given as

$$
\begin{aligned}
& a_{i-L}=E_{x}^{i}-L_{\text {min }}^{i-1}, \\
& a_{i-R}=L_{\max }^{i+1}-E_{x}^{i} .
\end{aligned}
$$

The second type of evaluation indicator is defined for minimum - optimum index; corresponding the lengths of left half-interval and right half-interval are given as

$$
\begin{aligned}
& a_{i-L}=E_{x}^{i}-L_{\min }^{i+1} \\
& a_{i-R}=L_{\max }^{i-1}-E_{x}^{i} .
\end{aligned}
$$

Certainty degree of evaluation indicators in each grade of evaluation sample is given according to (10); then combine indicator weights to calculate evaluation samples' integrated certainty degree to each class, shown as follows:

$$
\mu_{p, i}=\sum_{j=1}^{n} \mu_{p, i j} \lambda_{p, j}
$$

where $\mu_{p, i}$ is integrated certainty degree for the sample $p$ in grade $i$ and $\lambda_{p, j}$ is corresponding indicator weight.

The class is determined by the maximum integrated certainty degree; the criteria can be given as

$$
k=\max \left\{\mu_{p, 1}, \mu_{p, 2}, \ldots, \mu_{p, m}\right\},
$$

where $k$ is the evaluated class.

\section{Case Study}

In order to verify the validity and feasibility of this proposed model, the data of literature $[17,18]$ were used to conduct the analysis. The scale of debris flow, debris flow activity 
TABLE 1: Debris flow risk classification standard for evaluation indicators.

\begin{tabular}{lcccc}
\hline Category & Low risk (I) & Medium risk (II) & High risk (III) & Extremely high risk (IV) \\
\hline$C_{1} / 10^{4} \mathrm{~m}^{3}$ & $\leq 1$ & $1 \sim 10$ & $10 \sim 100$ & $\geq 100$ \\
$C_{2} / \%$ & $\leq 10$ & $10 \sim 50$ & $50 \sim 100$ & $\geq 100$ \\
$C_{3} / \mathrm{km}^{2}$ & $\leq 0.5$ & $0.5 \sim 10$ & $10 \sim 35$ & $\geq 35$ \\
$C_{4} / \mathrm{km}$ & $\leq 1$ & $1 \sim 5$ & $5 \sim 10$ & $\geq 10$ \\
$C_{5} / \mathrm{km}$ & $\leq 0.2$ & $0.2 \sim 0.5$ & $0.5 \sim 1.0$ & $\geq 1.0$ \\
$C_{6} /\left(\mathrm{km} / \mathrm{km}^{2}\right)$ & $\leq 5$ & $5 \sim 10$ & $10 \sim 20$ & $\geq 20$ \\
$C_{7}$ & $\leq 1.1$ & $1.1 \sim 1.25$ & $1.25 \sim 1.4$ & $\geq 1.4$ \\
$C_{8}$ & $\leq 0.1$ & $0.1 \sim 0.3$ & $0.3 \sim 0.6$ & $\geq 0.6$ \\
$C_{9} / \mathrm{mm}$ & $\leq 25$ & $25 \sim 50$ & $50 \sim 100$ & $\geq 100$ \\
$C_{10} /$ population $/ \mathrm{km}^{2}$ & $\leq 50$ & $50 \sim 150$ & $150 \sim 250$ & $\geq 250$ \\
& & & & \\
\hline
\end{tabular}

TABLE 2: Measured indicator values of samples.

\begin{tabular}{lcccccccccc}
\hline Sample & $C_{1}$ & $C_{2}$ & $C_{3}$ & $C_{4}$ & $C_{5}$ & $C_{6}$ & $C_{7}$ & $C_{8}$ & $C_{9}$ & $C_{10}$ \\
\hline 1 & 195.1 & 1500 & 47.10 & 12.00 & 2.19 & 23.8 & 1.45 & 0.8 & 102.0 & 260 \\
2 & 105.0 & 450 & 53.10 & 18.35 & 2.92 & 21.2 & 1.28 & 0.62 & 97 \\
3 & 7.8 & 20 & 10.61 & 4.61 & 1.66 & 12.8 & 1.12 & 0.40 & 100.4 & 210 \\
4 & 10.0 & 30 & 14.1 & 8.00 & 1.94 & 17.8 & 1.10 & 0.35 & 100.4 & 310 \\
5 & 1.8 & 80 & 4.20 & 2.10 & 1.19 & 12.3 & 1.11 & 0.42 & 100.4 & 318 \\
6 & 82.0 & 1200 & 18.05 & 11.8 & 1.66 & 22.8 & 1.39 & 0.72 & 100.4 & 0 \\
7 & 9.0 & 50 & 28.32 & 9.05 & 2.80 & 15.6 & 1.22 & 0.45 & 100.4 & 260 \\
8 & 5.0 & 50 & 3.28 & 2.30 & 1.13 & 22.0 & 1.28 & 0.78 & 98.0 & 276 \\
9 & 2.5 & 20 & 2 & 4.86 & 0.62 & 5.2 & 1.18 & 0.37 & 109.4 & 75 \\
10 & 3.2 & 25 & 4.23 & 5.08 & 0.67 & 5.3 & 1.14 & 0.33 & 109.4 & 80 \\
11 & 5.1 & 35 & 6.13 & 6.85 & 0.72 & 5.5 & 1.25 & 0.32 & 109.4 \\
12 & 2.5 & 35 & 3.02 & 5.6 & 0.61 & 4.5 & 1.28 & 0.3 & 109.4 \\
13 & 23 & 100 & 24.79 & 7.4 & 1.71 & 12.8 & 1.28 & 0.54 & 86.7 \\
14 & 0.4 & 20 & 0.15 & 1.13 & 0.23 & 8.2 & 1.14 & 0.29 & 69.2 \\
15 & 21 & 150 & 17.97 & 8.6 & 1.68 & 17.6 & 1.45 & 0.73 & 86.7 \\
\hline
\end{tabular}

frequency, watershed area, length of the main channel, the largest relative height of watershed, cutting density of watershed, bending coefficient of the main channel, length ratio of sediment supply, maximum $24 \mathrm{~h}$ rainfall, and population density of watershed were selected as the evaluation indicators. Risk grade was divided into low risk (I), medium risk (II), high risk (III), and extremely high risk (IV). The classification standard and the measured values of evaluation indexes were listed in Tables 1 and 2.

Based on the proposed model, corresponding digital feature values $\left(E_{x}, E_{n}, H_{e}, a^{\prime}, k\right)$ to each grade for evaluation indicators were calculated by (4) (8) and (11); then 2000 cloud drops of left half-interval and right halfinterval stimulated by a MATLAB program were obtained according to (3), so the asymmetric connection cloud mapping can be depicted from Figures $1(\mathrm{a})-1(\mathrm{j})$. To compare and analyze the reasonability of evaluation results, the weights of indexes were also from the literature [17]; $\lambda_{p, j}=\{0.22,0.23,0.11,0.09,0.08,0.08,0.02,0.06,0.07,0.04\}$. The evaluation results and comparison with other methods were listed in Table 3.

To better understand the whole calculation process of integrated certainty degree of measured samples, now take the indicator of bending coefficient of the main channel from the sample $2\left(x_{0}=1.28\right)$, for example, to illustrate evaluation process of debris flow risk grade.

First, the grade (I) of the seventh indicator can be determined for $[0,1.1]$ by the actual situation (the value of bending coefficient must not be less than 0 ), so the corresponding expected value $E_{x}=(0+1.1) / 2=0.5500$ by (4); then expected values of the grade (II) and (III) were determined for 1.1750 and 1.3250; the upper limitation value $L_{\text {mid }}=$ 1.55 of the grade (IV) was obtained by (9), so the expected value was 1.475 .

Then, the asymmetric connection cloud of grade (II) for the bending coefficient indicator was illustrated to calculate the corresponding certainty degree. The expected value $E_{x}$ of grade (II) had been calculated for 1.1750; then lengths of left half-interval $a_{i-L}$ and right half-interval $a_{i-R}$ were determined for 1.1750 and 0.2250 by (11). The orders of corresponding distribution density function $k_{i}$ in both left and right interval were 169.7823 and 5.8849 by (8).

Next, entropy $E_{n}$ and hyper entropy $H_{e}$ of left interval and right interval were 0.0635 and $0.0585,0.0006$, and 0.0006 by (5) and (6), respectively. 

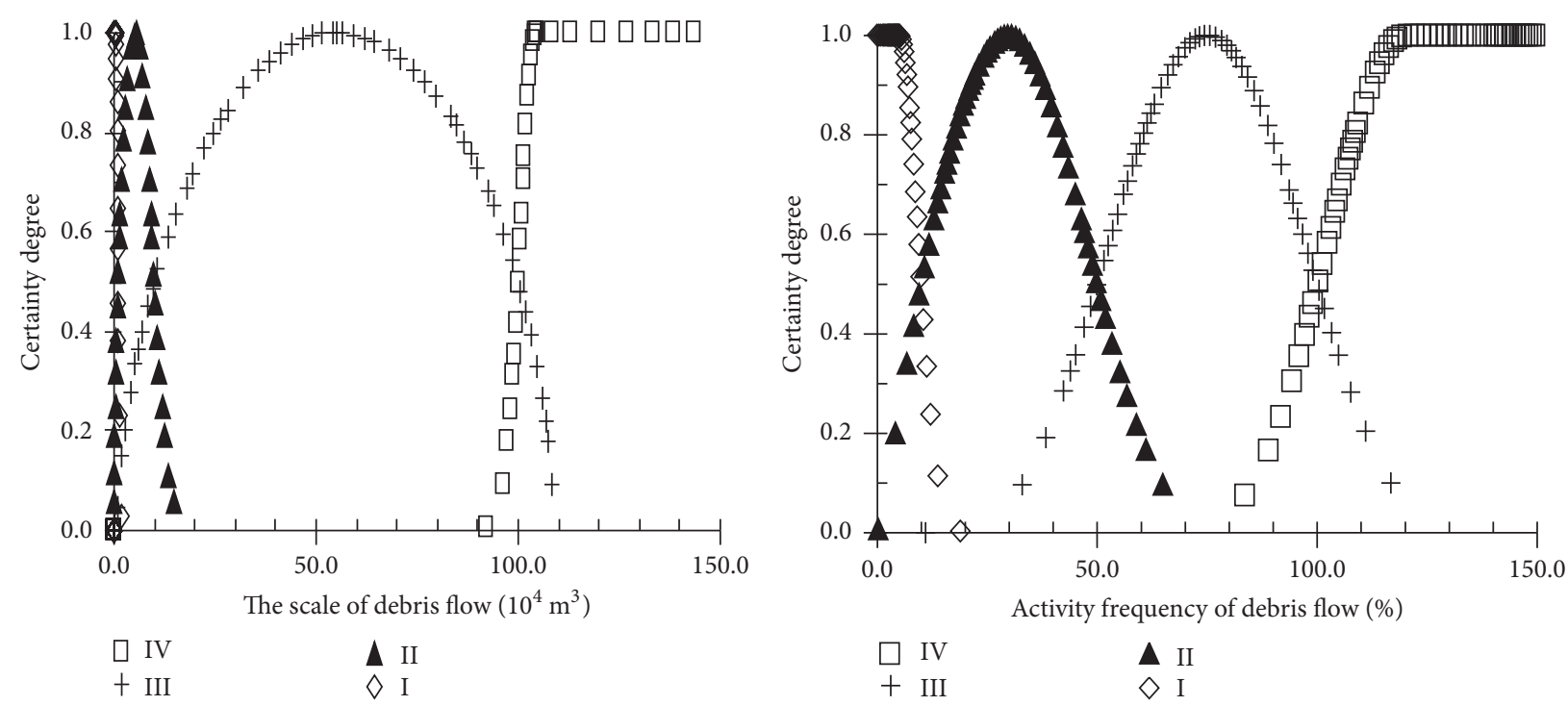

(a)

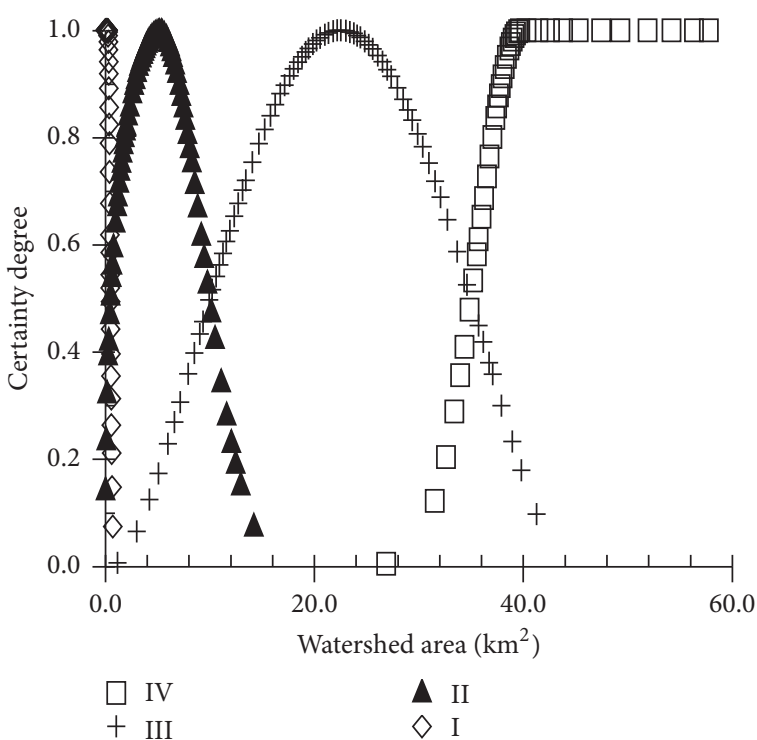

(c)
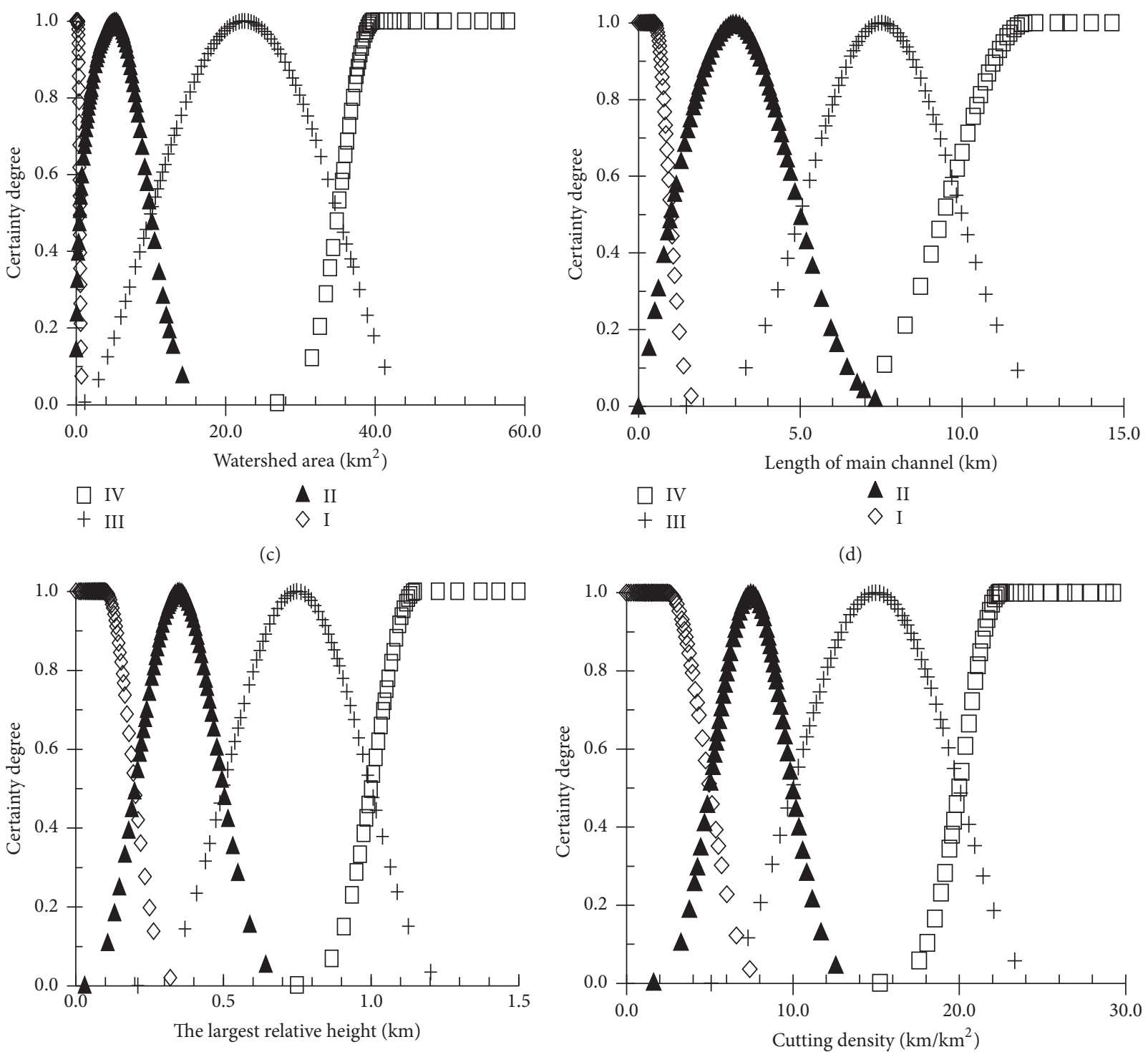

$\begin{array}{ll}\square \text { IV } & \boldsymbol{\Delta} \text { II } \\ + \text { III } & \diamond \text { I }\end{array}$

$\square$ IV

$\Delta$ II

(e)

(f)

FIgure 1: Continued. 


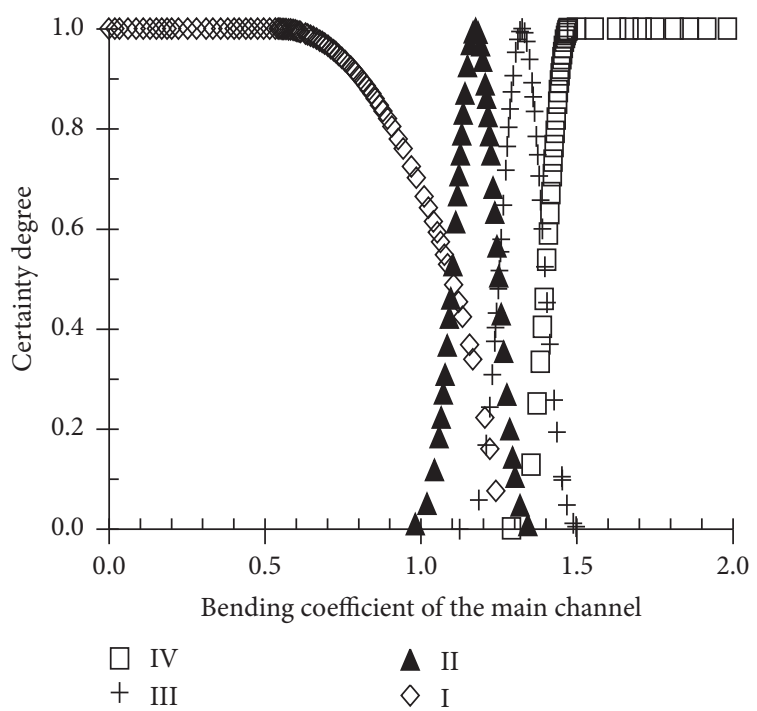

(g)

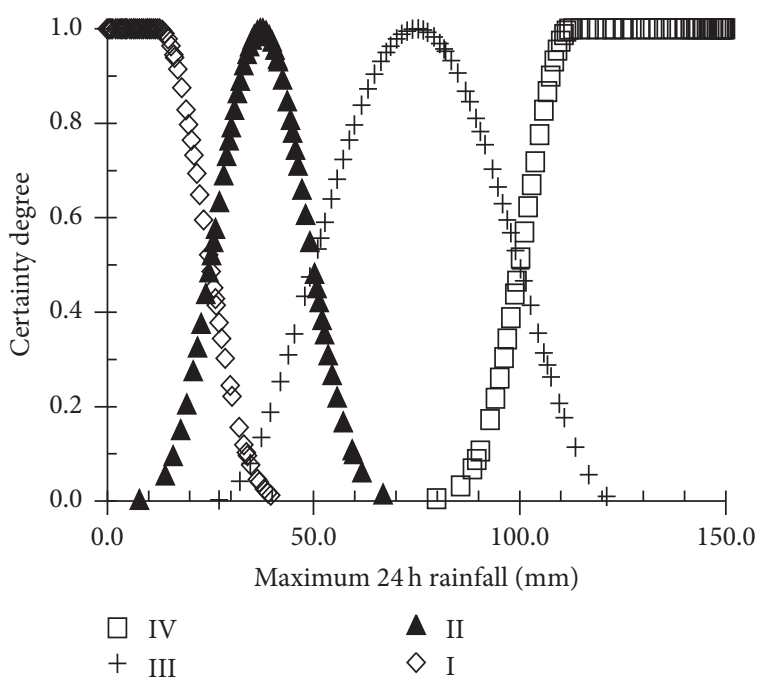

(i)

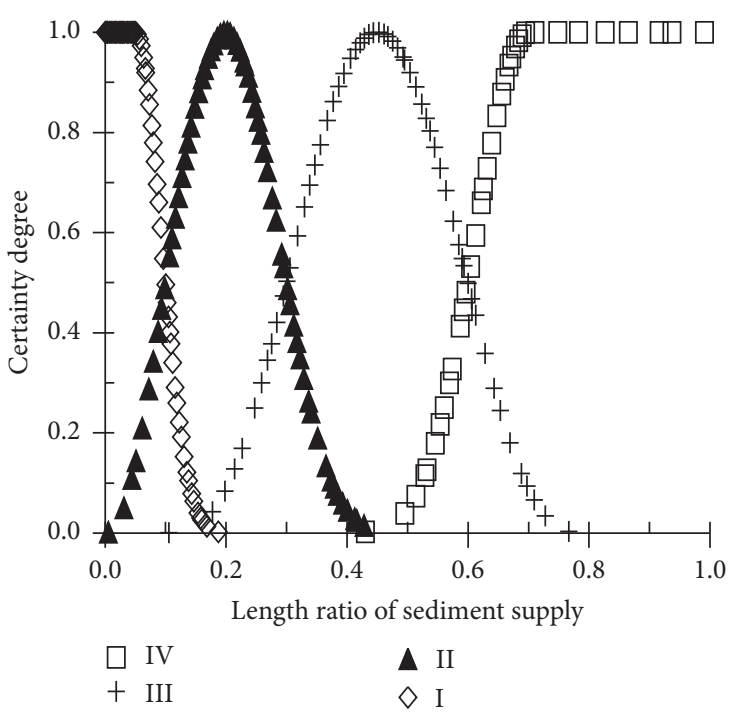

(h)

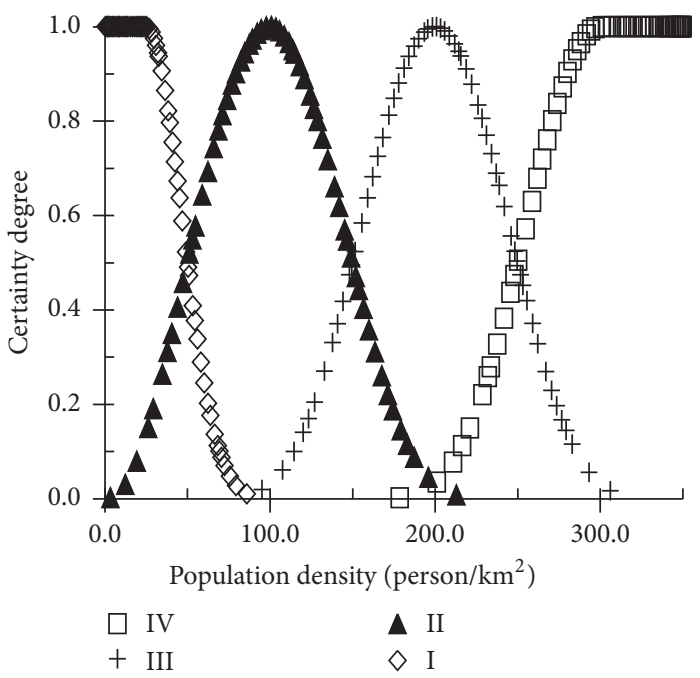

(j)

FIGURE 1: The asymmetric connection cloud mapping.

Then, next, the digital characteristic value $E_{n}^{\prime}$ of different interval was obtained for 0.0635 and 0.0578 according to the known $E_{n}$ and $H_{e}$.

Finally, the modified lengths of left half-interval $a_{i-L}^{\prime}$ and right half-interval $a_{i-R}^{\prime}$ were 1.1760 and 0.222 by (6). The corresponding asymmetric connection cloud mapping of grade (II) based on a MATLAB program was obtained by the cloud digital characteristic values and was shown as Figure 1(g). And other cloud mappings were also depicted by the same method. The certainty degree $\mu_{2,27}$ to the grade (II) for the 7 th index of sample 2 was determined for 0.2411 by (10), and certainty degree for other grades could also be calculated as $\mu_{2,17}=0, \mu_{2,37}=0.7919$, and $\mu_{2,47}=0.003$, respectively. The results indicated that the choice for the 7 th index of sample $2\left(x_{0}=1.28\right)$ determining risk grade (III) was the optimal; then the good choice was decided for risk grade (II) and the bad choice was defined for risk grade (IV), while the choice for risk grade (I) was the worst. The certainty degrees of the other indicators for vary grades were also determined; then combine the corresponding indicators weights and the integrated certainty degree was calculated by (13); the risk grade of measured sample was obtained by (14).

It was found from Table 3 that the results from the proposed model were almost same as those from the extension method and combined weights method and cloud model method. For sample 4 , there were five indicators whose measured values located in high grade, and only two indicators located in the medium grade; meanwhile weights of these five indicators are high, so it was more reasonable to specify it as high risk. Besides, for sample 5, the result of the proposed method was high risk, but medium risk by other methods. However, among measured evaluation index values for sample 5 , indicators for $C_{2}, C_{6}$, and $C_{8}$ were in high risk grade, and indicators for $C_{5}, C_{9}$, and $C_{10}$ were in extremely high risk 
TABLE 3: Integrated certainty degree of samples and comparison with other methods.

\begin{tabular}{|c|c|c|c|c|c|c|c|c|}
\hline \multirow{2}{*}{ Sample } & \multicolumn{4}{|c|}{ Integrated certainty degree } & \multirow{2}{*}{ Proposed model } & \multirow{2}{*}{ Combined weights method } & \multirow{2}{*}{ Extension method } & \multirow{2}{*}{ Cloud method } \\
\hline & $U(\mathrm{I})$ & $U(\mathrm{II})$ & $U$ (III) & $U(\mathrm{IV})$ & & & & \\
\hline 1 & 0.0000 & 0.0000 & 0.0555 & 0.9581 & IV & IV & IV & IV \\
\hline 2 & 0.0000 & 0.0052 & 0.2158 & 0.8621 & IV & IV & IV & IV \\
\hline 3 & 0.0096 & 0.4950 & 0.1533 & 0.1553 & II & II & II & II \\
\hline 4 & 0.0094 & 0.3996 & 0.4391 & 0.1771 & III & III & II & II \\
\hline 5 & 0.0020 & 0.3590 & 0.4278 & 0.1649 & III & II & II & III \\
\hline 6 & 0.0400 & 0.0005 & 0.3767 & 0.5835 & IV & IV & IV & IV \\
\hline 7 & 0.0027 & 0.2778 & 0.5770 & 0.1796 & III & III & III & III \\
\hline 8 & 0.0000 & 0.4409 & 0.2760 & 0.2792 & II & II & II & II \\
\hline 9 & 0.0378 & 0.6923 & 0.2308 & 0.0543 & II & II & II & II \\
\hline 10 & 0.0359 & 0.6890 & 0.2472 & 0.0543 & II & II & II & II \\
\hline 11 & 0.0351 & 0.6679 & 0.3590 & 0.0560 & II & II & II & II \\
\hline 12 & 0.0618 & 0.6258 & 0.2709 & 0.0540 & II & II & II & II \\
\hline 13 & 0.0000 & 0.0126 & 0.7172 & 0.2280 & III & III & III & III \\
\hline 14 & 0.3395 & 0.5543 & 0.1312 & 0.0299 & II & I & II & I \\
\hline 15 & 0.0000 & 0.0010 & 0.5194 & 0.4315 & III & III & III & III \\
\hline
\end{tabular}

grade. Therefore, measured indicator values of sample 5 were inclined to high risk, and it was relatively safe when it was specified as high risk not as medium risk. Also, for sample 14 , there were six indicators whose measured values located in medium risk grade, and indicator for $C_{9}$ was in high risk grade and indicator for $C_{10}$ was in extremely high risk grade, so it was more reasonable that sample 14 was determined for medium risk grade. The analyzed results indicated that the proposed method was feasible and more effective than other methods.

To compare and analyze asymmetric connection cloud and conventional cloud model, now take the indicator of maximum $24 \mathrm{~h}$ rainfall; for example, the results of cloud mapping based on conventional cloud model method and asymmetric connection cloud method were shown in Figure 2. It can be seen that the simulation value of indicator based on connectional cloud model may go beyond the range of measured value, more obviously in two-side interval, which is unreasonable with physical truth. Besides, the certainty degree of boundary value of two adjacent indicators according to conventional cloud model was vague and uncertain. However, the asymmetric connection cloud model can better avoid the above-mentioned drawbacks and also, combined with the IDC principle based on set pair theory, can convert the infinite interval of indicators distribution into finite interval, which makes the evaluation results more reliable and reasonable. And the certainty degree for 0.5 with boundary value of two adjacent indicators based on asymmetric connection cloud method can also be defined.

\section{Conclusions}

Risk evaluation of debris flow is a complicated and uncertainty problem since it is affected by various uncertainty factors. Based on measured values of evaluation indicators, the asymmetric connection cloud was discussed to analyze

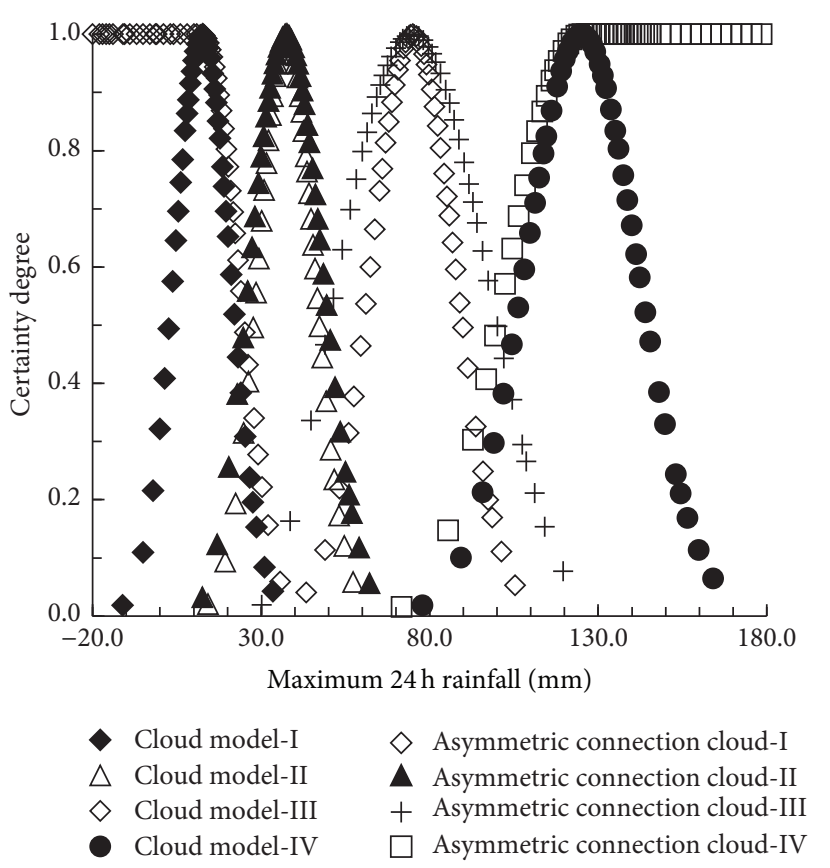

FIGURE 2: Comparison of cloud mapping between asymmetric connection cloud and cloud model.

the risk grade of debris flow in this paper. And case study and comparison with the extension method and combined weights method were also conducted. The results indicated that this proposed method can take the set pair analysis theory into consideration for depicting the asymmetric connection cloud mapping and can quantitatively describe certainty and uncertainty relationship between evaluation indicators and classification standard in a unified way. Besides, it can avoid the limitation for index definitely in the form of normal distribution in the conventional normal cloud and 
also convert the infinite interval of indicators distribution into finite interval and analyze dialectically the uncertainty of evaluation indicators, which enhances the reasonability of risk assessment of debris flow, and it also is useful in the similar evaluation problems.

\section{Conflicts of Interest}

The authors declare that they have no conflicts of interest.

\section{Acknowledgments}

Financial support provided by the National Key Research and Development Program of China under Grant no. 2016YFC0401303 and the National Natural Sciences Foundation, China (nos. 41172274 and 71273081) is gratefully acknowledged.

\section{References}

[1] P. Cui, "Advances in debris flow prevention in China," Science of Soil and Water Conversation, vol. 7, pp. 7-13, 2009 (Chinese).

[2] T. Glade, "Linking debris-flow hazard assessments with geomorphology," Geomorphology, vol. 66, pp. 189-213, 2005.

[3] Y. Q. Yang, J. F. Hu, and M. H. Xi, "Analysis of, influence of debris flow disaster in ZhouQu based on grey relational analysis method," Journal of Natural Disasters, vol. 3, pp. 139-144, 2011 (Chinese).

[4] Z. Yang, Y. Zhu, D. H. S. Zou, and L. Liao, "Activity degree evaluation of glacial debris flow along international Karakorum Highway (KKH) based on fuzzy theory," Advanced Materials Research, vol. 261-263, pp. 1167-1171, 2011.

[5] Y. Kuang, L. R. Xu, and B. C. Liu, "Debris flow risk assessment based on extension method," Journal of China Railway Science, vol. 27, no. 5, pp. 1-6, 2006 (Chinese).

[6] G. Z. Luo, L. Xu, and R. Xu, "Hazard assessment to debris flow to highway of mountain area-application of stratification analysis method to extension theory," Journal of Geological Hazards and Environment Preservation, vol. 19, pp. 128-133, 2008 (Chinese).

[7] Y. Liu, H. C. Guo, R. Zou, and L. J. Wang, "Neural network modeling for regional hazard assessment of debris flow in Lake Qionghai Watershed, China," Environmental Geology, vol. 49, no. 7, pp. 968-976, 2006.

[8] J. G. Santos, "GIS-based hazard and risk maps of the Douro river basin (north-eastern Portugal)," Geomatics, Natural Hazards and Risk, vol. 6, pp. 1-25, 2013.

[9] Y. P. He, H. Xie, P. Cui, F. Q. Wei, D. L. Zhong, and J. S. Gardner, "GIS-based hazard mapping and zonation of debris flows in Xiaojiang Basin, southwestern China," Environmental Geology, vol. 45, no. 2, pp. 286-293, 2003.

[10] D. Y. Li, Artificial Intelligence with Uncertainty, National Defence Industry Press, Beijing, China, 2005 (Chinese).

[11] K. Q. Zhao, Set Pair Analysis and Its Preliminary Application, Zhejiang Science and Technology Press, HangZhou, China, 2000 (Chinese).

[12] W.-S. Wang, J. L. Jin, J. Ding, and Y. Q. Li, "A new approach to water resources system assessment-set pair analysis method," Science China Technological Sciences, vol. 52, no. 10, pp. 30173023, 2009.
[13] M. W. Wang, J. L. Jin, and Y. L. Zhou, Set Pair Analysis Based Coupling Methods and Applications, Science Press, BeiJing, China, 2014 (Chinese).

[14] M. Wang and G. Chen, "A novel coupling model for risk analysis of swell and shrinkage of expansive soils," Computers \& Mathematics with Applications, vol. 62, no. 7, pp. 2854-2861, 2011.

[15] M.-W. Wang, P. Xu, J. Li, and K.-Y. Zhao, "A novel set pair analysis method based on variable weights for liquefaction evaluation," Natural Hazards, vol. 70, no. 2, pp. 1527-1534, 2014.

[16] M. Wang, X. Xu, J. Li, J. Jin, and F. Shen, "A novel model of set pair analysis coupled with extenics for evaluation of surrounding rock stability," Mathematical Problems in Engineering, vol. 2015, Article ID 892549, 9 pages, 2015.

[17] Y. Lu, L. Xu, S. Chen, and L. Cao, "Combined weight method based on game theory for debris flow hazard risk assessment," Journal of Catastrophology, vol. 29, pp. 194-200, 2014 (Chinese).

[18] X. L. Liu and C. Tang, Risk Assessment of Debris Flow, Science Press, 1995 (Chinese). 


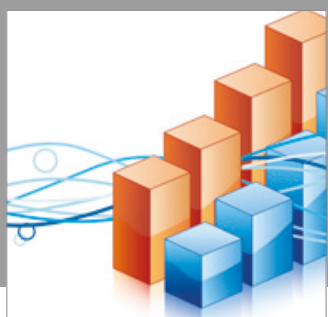

Advances in

Operations Research

vatersals

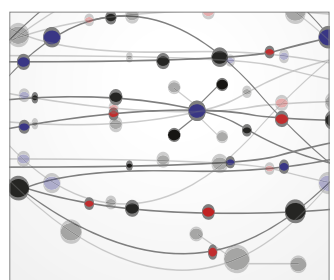

\section{The Scientific} World Journal
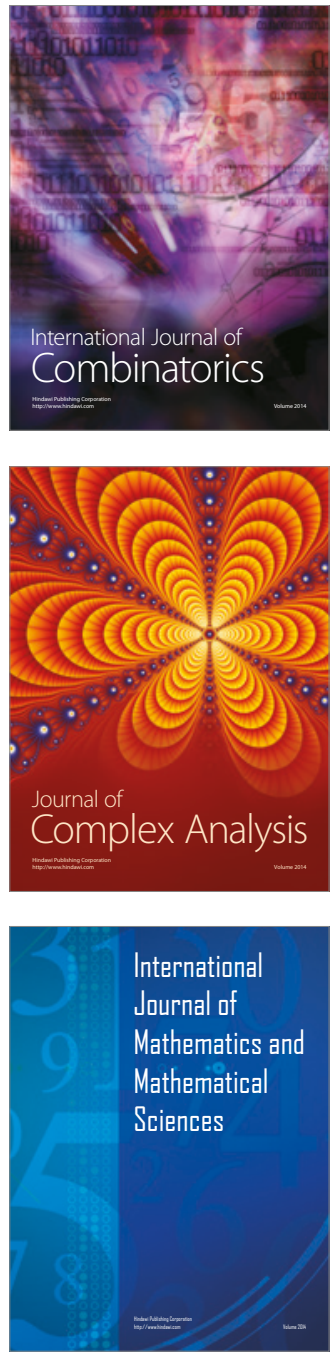
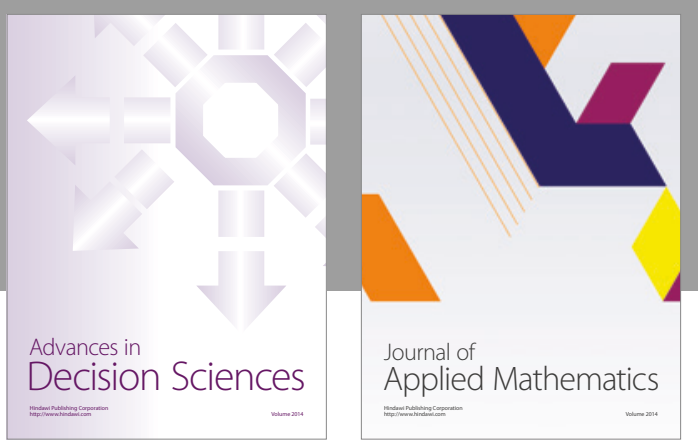

Algebra

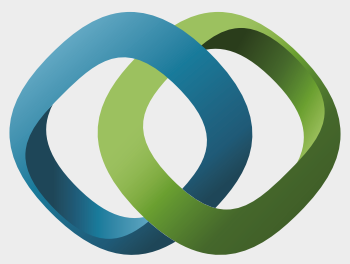

\section{Hindawi}

Submit your manuscripts at

https://www.hindawi.com
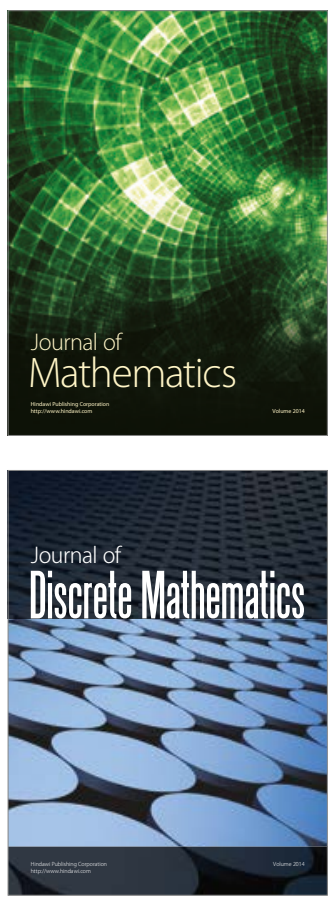

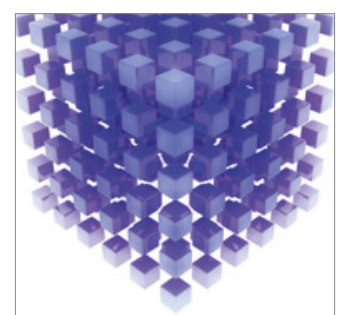

Mathematical Problems in Engineering
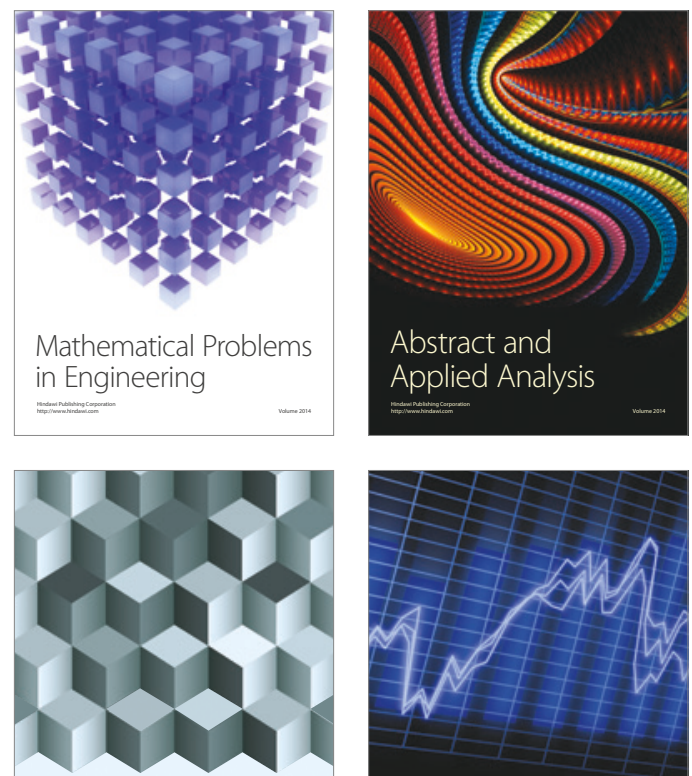

Journal of

Function Spaces

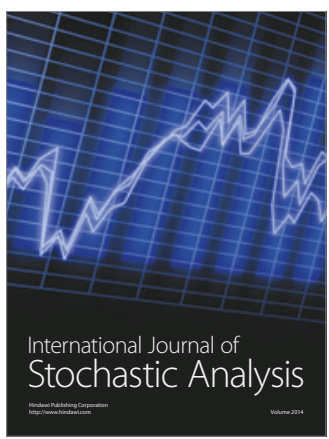

Probability and Statistics
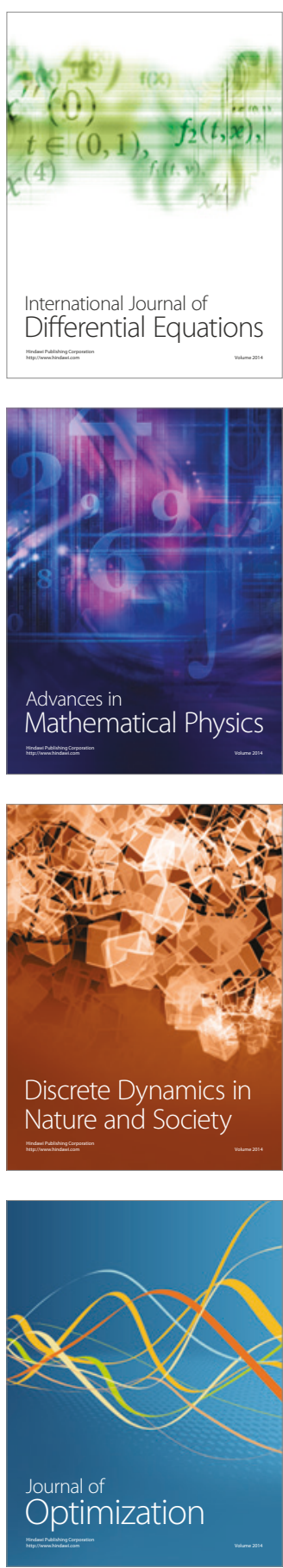